\title{
Improved Electric Power Demand Forecasting by adapting the Weighted Average to the MISMO Strategy
}

\author{
TCHUIDJAN Roger, HAMANDJODA Oumarou, MBOBDA Gérard, TABE Moses, \\ TOUKE Yannick \\ Power Unit - Energetics and Automation Laboratory \\ National Advanced School of Engineering - University of Yaounde I - Cameroon \\ tchuir@yahoo.fr
}

\begin{abstract}
Forecasting the annual long-term consumption of electrical energy in a country has remained for the Electrical Engineer quite a difficult problem to solve. As an important planning tool, the forecast of electrical energy consumption has to be as precise as possible. The most commonly employed method is that of scenario building. With the scenario method, consumption forecasting is done through the simulation of a sequence of events. The generated data cannot therefore be as stochastic as it is in reality. With the advent of the computer age, numerous other statistical methods for consumption forecasting have been developed. Prominent among them is the forecasting by machine learning with multiple-Input multiple-Output local learning strategy. The objective here is to obtain a forecast which is as precise as possible, while conserving the stochastic nature between the historical and the forecasted data.

This article first presents the different strategies for long-term power consumption forecasting using Multiple-Input Multiple-Output local learning strategies. It then proposes, based on the work of earlier researchers, an approach that uses the weighted averages to improve on the level of precision obtained. Furthermore, it applies this new improved calculation method to forecast the power consumption specifically in Cameroon for horizon 2035, when the country aspires to become an emerging economy. The last part of this article utilizes historical data on the electricity consumption of some countries from the World Bank dataset to do a comparative study between the here newly proposed method and that used previously. The results show that, the new method using MISMO plus weighted average delivers more exact results for long-term electrical power consumption forecasts.
\end{abstract}

Keywords: long-term forecasting, automatic apprenticeship, nearest neighbors, weighted average, electric energy consumption

\section{Introduction}

Long-term annual electricity consumption forecasting is an important tool for major decision-makers like governments and multinational investors. It serves not only in the planning of power supply for future investments, but also serves in the scheduling of generation and transmission equipment maintenance. Ideally, an investor would want to know the exact future demand. Since this is impossible, only long-term electrical demand forecast methods can be used to estimate future annual electricity consumptions. It is therefore evident that such long-term forecasts have to come as close as possible to reality.

For the past years, many research endeavors have not only developed long-term electricity annual load demand forecast methods, but also improved on their level of accuracy. One of the most popular methods used for this purpose is that called "scenarios building". EDF used the scenarios building method to forecast the annual electricity consumption of France from 2012 to 2020. The same method was used in [11] to forecast Cameroon's electricity demand from 2005 to 2030. Also, [19] employed this method to do a forecast of Tahiti's annual electricity demand from 2005 to 2020. This method is known to be not quite realistic and somewhat illusionistic too. To overcome these weaknesses, quantitative methods known as time series-

Received: August $30^{\text {th }}, 2012$. Accepted: March $28^{\text {th }}, 2014$ 
based methods were introduced. One of the most popular of the time series-based methods used for this purpose is the Autoregressive Moving Average (ARMA). For instance, Edigera and Akarb [5] used seasonal ARIMA (SARIMA) to estimate the future primary energy consumption of Turkey from 2005 to 2020. Biancoa et al [2] also proposed a linear regression model for Italy's electricity consumption.

However, such methods, generally referred to as linear methods, can give accurate forecasts if and only if there is some linearity in the past data. They therefore fail to capture data with non- linearities. With the advent of the computer, Computer Intelligence (CI)-based methods have been at the center of many research undertakings in the area of long-term annual electricity demand forecasting. The main advantage of these methods is that they can capture both linear and non-linear behavior of the time series. Machine learning, Fuzzy logic and Artificial Neural Network (ANN) are three main CI-based techniques, which have shown how powerful they are in capturing both linearity and non-linearity in data. Hossein Iranmanesh et al [7] proposed a Mid-Term Energy demand forecasting using hybrid Neuro-Fuzzy models. Their method was used to estimate the U.S monthly gasoline demand in 2010. In their study, they combined Local Linear Neuro-Fuzzy (LLNF) models and Hodrick-Prescott (HP) filter and showed how powerful the HP filter was in accuracy improvement. J.V Ringwood and D. Bofelo proposed an approach of short (hourly), medium (weekly) and long-term (yearly) electricity demand forecasting using neural networks [8]. A combination of neural networks and fuzzy inference systems was proposed by Chen for long-term forecasting of the electrical load in Taiwan [18]. They were preceded by Nuorati et al, who also proposed a Neuro-Fuzzy model for long-term electrical load forecasting [13]. Souhaib Ben Taieb et al combined direct and MIMO strategies (MISMO) and developed a new long-term prediction method for time series [16]. Their proposed strategy was tested on the European Symposium for Time Series Prediction (ESTSP) competition in 2007. It came second. Many other methods have been proposed by different researchers as mentioned by the above authors. For example, [7] mentioned the studies of Han et al, who propose a review on electric load forecasting. They have provided an overview on classical time series and regression methods, as well as artificial intelligence and computational intelligence approaches [7]. However, very little has been done in the field of annual long-term electricity consumption.

In this paper, it is shown that the accuracy of the original MISMO strategy [16] can be improved when combining it to the Weighted Average [17]. Then this method is used to forecast the annual Cameroonian electricity consumption from 2012 to 2035. The rest of the paper is organized as follows: Section II briefly recalls the MISMO strategy methodology followed by the weighted average method. Section III describes our proposed methodology to improve the accuracy of the MISMO strategy and section IV gives some comparative results on the forecasting of the annual electricity demand for some countries.

\section{The MISMO Strategy}

In this section the MISMO strategy (Multiple Input Several Multiple Outputs) shall be briefly described.

Firstly, an overview of long-term prediction techniques using machine learning is necessary. Considering a univariate time series $\left\{Y_{1}, Y_{2}, \ldots, Y_{N}\right\}$ for which the next $\mathrm{H}$ values need to be predicted, there are two main strategies that can be adopted: the direct strategy and the recursive strategy.

\section{A. The Direct Strategy}

The first strategy initially embeds the time series into $\mathrm{H}$ datasets as follows: 


$$
\begin{gathered}
D_{1}=\left\{\left(\mathbf{x}_{i 1}, y_{i 1}\right) \in\left(\mathbf{R}^{d} \mathbf{x} \mathbf{R}\right)\right\}_{i=1}^{N} \\
\vdots \\
D_{H}=\left\{\left(\mathbf{x}_{i H}, y_{i H}\right) \in\left(\mathbf{R}^{d} \mathbf{x} \mathbf{R}\right)\right\}_{i=1}^{N}
\end{gathered}
$$

With $d$ the maximum embedded order, and

$$
\begin{aligned}
& \mathbf{x}_{i h}=\left\{Y_{i, \ldots,}, Y_{i+d-1}\right\} \\
& y_{i h}=\left\{Y_{i+d-1+h}\right\}, h \in\{1, \ldots, H\}
\end{aligned}
$$

Then $\mathrm{H}$ models $f_{h}($.$) are trained using the datasets in order to obtain the \mathrm{H}$ predictions as follows:

$$
y_{i h}=f_{h}\left(\mathbf{x}_{i h}\right)+w_{i h}
$$

Where $w_{i h}$ is an additive noise

B. The Recursive Strategy

In contrast, the recursive strategy after embedding the time series is as follows:

$$
\begin{aligned}
& D=\left\{\left(\mathbf{x}_{i}, y_{i}\right) \in\left(\mathbf{R}^{d} \mathbf{x} \mathbf{R}\right)\right\}_{i=1}^{N} \\
& \mathbf{x}_{i}=\left\{Y_{i}, \ldots, Y_{i+d-1}\right\} \\
& y_{i}=\left\{Y_{i+d}\right\}
\end{aligned}
$$

The dataset is used to train a function which estimates the next value of the series as follows:

$$
Y_{N+1}=f\left(\mathbf{x}^{*}\right)
$$

where $\mathbf{x}^{*}=\left\{Y_{N-d+1}, \ldots, Y_{N}\right\}$

Then the estimated value is used as an input to predict the following value as follows:

$$
\underline{Y}_{N+2}=f\left(\left\{\mathbf{x}^{*} / Y_{N-d+1}, Y_{N+1}\right\}\right)
$$

And the next values are estimated in the same manner.

Usually, in order to obtain accurate forecasts, an input variable selection algorithm is used to choose the best subsets between the input variables.

The recursive strategy suffers from error accumulation while the direct strategy suffers from the strong conditional independence of the trained models. The multiple input multiple output (MIMO) strategy was introduced to combine the advantages of both the direct and the recursive strategies [16], reduce the error due to the recursive method and remove the conditional independence of the direct strategy.

C. The MIMO Strategy

The MIMO strategy embeds the dataset into a multidimensional input output variable set as follows:

$$
D_{i}=\left\{\left(\mathbf{x}_{i}, \mathbf{y}_{i}\right) \in\left(\mathbf{R}^{d} \times \mathbf{R}^{H}\right)\right\}_{i=1}^{N}
$$

Where

$$
\begin{aligned}
& \mathbf{x}_{i}=\left\{Y_{i}, \ldots, Y_{i+d-1}\right\} \\
& \mathbf{y}_{i}=\left\{Y_{i}, \ldots, Y_{i+d+H-1}\right\}
\end{aligned}
$$

Then a multidimensional function $f($.$) is trained from the dataset as follows:$ 


$$
\left\{\mathbf{y}_{i}\right\}=f\left(\mathbf{x}_{i}\right)+w_{i}
$$

Then the function is used to predict the next $\mathrm{H}$ values.

However this method constrains all the horizons to be predicted with the same model structure, thus reducing the flexibility of the MIMO strategy [16].

\section{The MISMO Strategy}

The MISMO strategy was introduced by [16] in order to resolve the problem of flexibility imposed by the MIMO strategy. The MISMO strategy brings the problem of prediction of horizon $\mathrm{H}$ into $\mathrm{n}$ prediction part problems of horizon $\mathrm{s}$. That is, a problem of prediction of horizon $\mathrm{H}$ is broken down into several problems of prediction of horizon s. This is why it is called multiple input several multiple outputs (MISMO). The time series is therefore divided into $\mathrm{n}$ portions of training datasets as follows:

$$
\begin{gathered}
D_{1}=\left\{\left(\mathbf{x}_{i 1}, \mathbf{y}_{i 1}\right) \in\left(R^{d} \times R^{s}\right)\right\}_{i=1}^{N-d-H} \\
\vdots \\
D_{n}=\left\{\left(\mathbf{x}_{\text {in }}, \mathbf{y}_{\text {in }}\right) \in\left(R^{d} \times R^{s}\right)\right\}_{i=1}^{N-d-H}
\end{gathered}
$$

Where

$$
\begin{aligned}
& \mathbf{x}_{i p}=\left\{Y_{i}, \ldots, Y_{i+d-1}\right\}, \\
& \mathbf{y}_{i p}=\left\{Y_{i+d+(p-1) s}, \ldots, Y_{i+d+p s-1}\right\}, p=\{1, \ldots, n\} \\
& n=\frac{H}{s}
\end{aligned}
$$

The MISMO strategy therefore trains $\mathrm{n}$ models $f_{p}($.$) with$

$$
\left\{\mathbf{y}_{i p}\right\}=f_{p}\left(\mathbf{x}_{i p}\right)+\mathbf{w}_{i p}
$$

Then each model is used to estimate the s desired predictions in the following manner:

$$
\begin{gathered}
\left\{Y_{t+1}, \ldots, Y_{t+(s-1)}\right\}=f_{1}\left(\mathbf{x}^{*}\right), \\
\left\{Y_{t+s}, \ldots, Y_{t+(2 s-1)}\right\}=f_{2}\left(\mathbf{x}^{*}\right) \\
\vdots
\end{gathered}
$$

\section{E. Learning and Validation}

Learning and validation are two terms commonly used in machine training. The former is the process through which information is extracted from data, while validation is the process through which the performance of a model is estimated [7], [16].

The estimation of the functions $f_{p}($.$) is based on the nearest neighbor approach. In order$ to adjust the number of nearest neighbors, a lazy learning algorithm is used [16]. The general methodology to forecast using the MISMO strategy is as follows:

\section{Given a query point $\mathbf{x}^{*}$}

- Select the best subset of inputs $\mathbf{x}_{i} \subset\left\{Y_{i}, \ldots, Y_{i+d-1}\right\}$ which can best predict the desired value

- Determine the optimal value of $s$ 
- Divide the horizon of prediction into nH into n portions where $n=\frac{H}{\mathrm{~S}}$ as in (13)

- For each input $\mathbf{x}_{i}$ calculate the Euclidean distance from the query point $\left(\left\|\mathbf{x}_{i}-\mathbf{x}^{*}\right\|\right)$ in order to determine the kk nearest neighbors. The kk nearest neighbors are the variables that give the smallest value of the calculated Euclidean distance.

- For each portion, the output is estimated as:

$\hat{\mathbf{y}}_{q p}=\frac{1}{k} \sum_{j=1}^{k} y_{[j] p}$

Where

$y_{[j] p}$ is the output of the $j$ th closest neighbor of $\mathbf{x}^{*}$ in the dataset $D_{p}$

- Concatenate these outputs to obtain the prediction as follows:

- $\hat{\mathbf{y}}_{q}=\left\{\hat{\mathbf{y}}_{q 1}, \hat{\mathbf{y}}_{q 2}, \ldots, \hat{\mathbf{y}}_{q n}\right\}$

- Estimate the performance of the model using chosen criteria. [16] proposed two methods to estimate the performance of the model, one based on cross validation and the other based on a measure of stochastic discrepancy between the forecasted sequence and the training time series.

- Choose the best model depending on the desired criteria.

The algorithms of each step mentioned above are developed in [4], [16].

\section{F. Weighted Moving Average}

The weighted moving average is introduced by [17] to estimate the future value of a time series. For the above time series, the future value can be estimated by the weighted moving average as follows:

$$
Y_{N+1}=\sum_{j=-m}^{m} \alpha_{j} Y_{N+1+j}
$$

Where

$m=\frac{k-1}{2}$

$\alpha_{j}$ are the weights associated to each value

$k$ the number of observations used to predict the output

A variety of weight functions is developed by [17]. The advantage of this method is that the influence of each observation to the predicted output can be modified by adjusting the corresponding weight. Thus, the higher the weight the more important is the corresponding observation in predicting the output. Usually, the sum of the weights is normalized to one.

\section{The Proposed Methodology}

In this section, the new methodology is presented. This methodology combines the MISMO strategy and the Weighted Moving Average. The MISMO strategy forecasts the next values of the time series by averaging the outputs of the nearest neighbors of the query. This assumes that, the output of each neighbor is considered having the same influence in the forecast, which therefore tends to diminish the strong hypothesis that underlies the nearest neighbor theory. The nearest neighbor theory assumes that, if two random variables $X$ and $Y$ are considered, and supposing that $X=Y$, then for a given function $f($.$) :$ 


$$
f(X)=f(Y)
$$

This also means that outputs of the points that are nearer to the query point have more information concerning the predicted values.

To take this fact into consideration, this paper therefore proposes that after determining the $\mathrm{k}$ nearest neighbors, the outputs should be estimated using a weighted average. The weights should decrease with an increase in the distance from the query point. In this paper the following weight function is proposed:

$$
\alpha_{j}=\frac{\frac{1}{d_{j}}}{\sum_{i=1}^{k} \frac{1}{d_{i}}}
$$

Where

$j$ represents the $j$ th nearest neighbor of the query point $\mathbf{x}^{*}$

$d_{j}$ the euclidean distance from $\mathbf{x}_{j}$ to $\mathbf{x}^{*}$ calculated as $\left\|\mathbf{x}_{i}-\mathbf{x}^{*}\right\|$

The term $\sum_{i=1}^{k} \frac{1}{d_{i}}$ is used to normalize the weights

Therefore, the nearer an input to the query, the smaller is the distance and higher is the corresponding weight. In this way, the contribution of each neighbor is proportional to the information it contains about the query point.

After estimating the weight functions in this way, the corresponding Leave One Out error has to be re-estimated. With the corresponding weight function, the Leave One error then becomes:

$$
\begin{aligned}
e_{j}(k)=y_{j}-\sum_{i=1, i \neq j}^{k}\left(\frac{\frac{1}{d_{i}} y_{i}}{\sum_{i=1, i \neq j}^{k} \frac{1}{d_{i}}}\right) \\
=y_{j}-\frac{\sum_{i=1, i \neq j}^{k} \frac{1}{d_{i}} y_{i}}{\sum_{i=1, i \neq j}^{k} \frac{1}{d_{i}}} \\
=\left(\sum_{i=1}^{k} \frac{1}{d_{i}}\right) *\left(\frac{y_{i}-\hat{y}_{k}}{\sum_{i=1, i \neq j}^{k} \frac{1}{d_{i}}}\right)
\end{aligned}
$$

Which finally gives:

$$
e_{j}(k)=\left(\sum_{i=1}^{k} \frac{1}{d_{i}}\right) *\left(\frac{y_{i}-\hat{y}_{k}}{\sum_{i=1, i \neq j}^{k} \frac{1}{d_{i}}}\right)
$$

It is seen from (23) and (24) that setting the $d_{i}$ to one (or to the same values) leads to (19) 


\section{Evaluation of the New Method on Data Sets}

The performance of the new method was tested by forecasting the annual electricity consumption of Cameroun, China, Brazil, India, South Africa, France, Germany, USA and Italy. The model is selected based on the minimum error criteria, and not the minimum average criteria. The data used was obtained from the World Bank dataset.

The dataset set consists of values from 1971 to 2009 for each country except, for that of the USA, Italy, France and Germany which consists of values from 1960 to 2009. The data for each country will be divided into two parts: one for training and the other for testing. In order to appreciate the method, the forecasts for $\mathrm{H}=1,2,3,10$ and 15 are done. After what, the Cameroonian electricity consumption from 2012 to 2035 is forecasted using the newly proposed method. Figure 1 below shows some data used:
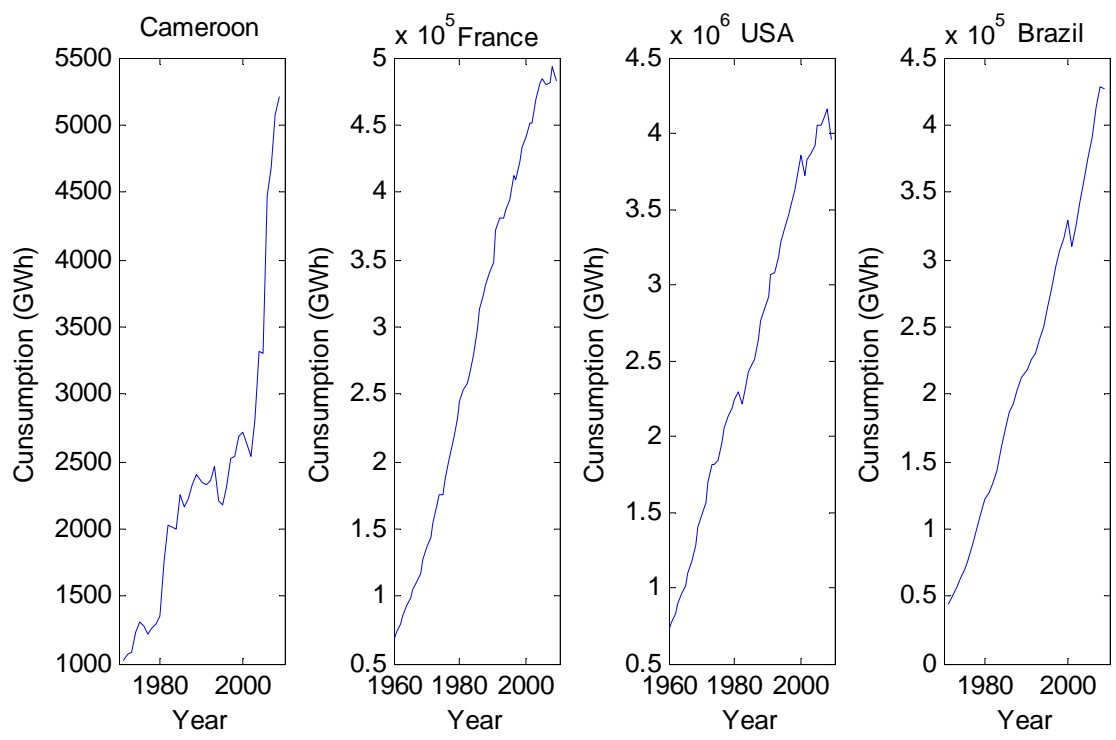

Figure 1. Examples of data used

As shown by the figure, most of the time series contain a trend pattern. Therefore, the time series are firstly de-trended as recommended by the MISMO strategy using the Mann Kendall test [3].

The embedded value is chosen to one since values are collected annually.

\section{Results and Discussions}

The newly proposed methodology was compared to the original MISMO_CV strategy using the Leave One out algorithm. The comparison was done using two statistical quantities, namely the Mean Absolute Percentage Error (MAPE) [16]:

$$
M A P E=\frac{1}{H} \sum_{i=1}^{H}\left|\left(\frac{Y_{t+i}-Y_{t+i}}{Y_{t+i}}\right) \times 100\right|
$$

Where

\section{$Y_{t}$ represents the forecasted value at time $\mathrm{t}$}

\section{$H$ the horizon of prediction}

And the Symmetric Mean Absolute Percentage Error (SMAPE) [16] 
TCHUIDJAN Roger, et al.

$$
\text { SMAPE }=\frac{1}{H} \sum_{i=1}^{H}\left[\frac{\left|Y_{t+i}-Y_{t+i}\right|}{\frac{Y_{t+i}+Y_{t+i}}{2}} * 100\right]
$$

Then the MAPE and the SMAPE for each horizon are averaged to obtain a mean MAPE and SMAPE. The table below summarizes the results obtained

Table 1. SMAPE and MAPE for the two strategies

\begin{tabular}{|c|c|c|c|c|c|c|c|c|c|c|c|}
\hline \multicolumn{3}{|c|}{ Country } & Germany & USA & Italy & France & $\begin{array}{l}\text { South- } \\
\text { Africa }\end{array}$ & Cameroon & Brazil & India & China \\
\hline \multirow{20}{*}{$\begin{array}{l}\text { 음 } \\
\text { 을 } \\
\text { 어 }\end{array}$} & \multirow{4}{*}{1} & Smape 1 & 4.04 & 6.18 & 8.08 & 2.92 & 5.85 & 4.96 & 4.68 & 3.38 & 8.28 \\
\hline & & Smape 2 & 4.01 & 6.19 & 8.33 & 3.77 & 5.92 & 6.27 & 5.15 & 3.69 & 7.60 \\
\hline & & Mape 1 & 4.12 & 6.38 & 8.42 & 2.96 & 6.10 & 5.09 & 4.79 & 3.44 & 7.96 \\
\hline & & Mape 2 & 4.09 & 6.38 & 8.69 & 3.84 & 6.03 & 6.45 & 5.28 & 3.76 & 7.32 \\
\hline & \multirow{4}{*}{2} & Smape 1 & 4.42 & 3.87 & 6.17 & 1.80 & 8.42 & 1.48 & 3.88 & 7.12 & 11.55 \\
\hline & & Smape 2 & 3.79 & 3.81 & 6.17 & 1.81 & 8.74 & 1.32 & 3.91 & 6.13 & 11.82 \\
\hline & & Mape 1 & 4.58 & 3.99 & 6.45 & 1.82 & 8.85 & 1.48 & 4.00 & 7.45 & 12.32 \\
\hline & & Mape 2 & 3.92 & 3.93 & 6.47 & 1.83 & 9.19 & 1.33 & 4.04 & 6.36 & 12.63 \\
\hline & \multirow{4}{*}{3} & Smape 1 & 2.72 & 3.66 & 5.73 & 2.90 & 5.84 & 7.79 & 2.15 & 2.70 & 9.48 \\
\hline & & Smape 2 & 2.09 & 3.97 & 5.26 & 2.41 & 5.58 & 8.60 & 2.29 & 2.33 & 9.85 \\
\hline & & Mape 1 & 2.79 & 3.80 & 5.99 & 2.96 & 6.13 & 7.45 & 2.16 & 2.76 & 10.21 \\
\hline & & Mape 2 & 2.05 & 4.12 & 5.47 & 2.46 & 5.84 & 8.17 & 2.29 & 2.37 & 10.63 \\
\hline & \multirow{4}{*}{10} & Smape 1 & 3.25 & 5.10 & 5.45 & 3.83 & 2.74 & 21.29 & 4.82 & 5.65 & 8.94 \\
\hline & & Smape 2 & 3.78 & 5.85 & 5.15 & 2.01 & 1.96 & 22.45 & 4.05 & 6.32 & 12.10 \\
\hline & & Mape 1 & 3.34 & 5.27 & 5.76 & 3.98 & 2.74 & 19.20 & 4.73 & 5.41 & 8.42 \\
\hline & & Mape 2 & 3.74 & 6.06 & 5.46 & 2.05 & 2.02 & 18.97 & 4.01 & 6.01 & 11.16 \\
\hline & \multirow{4}{*}{15} & Smape 1 & 11.69 & 2.07 & 3.09 & 6.23 & 4.07 & 16.33 & 3.92 & 12.18 & 16.76 \\
\hline & & Smape 2 & 8.38 & 3.43 & 2.15 & 7.80 & 4.67 & 16.80 & 6.57 & 16.31 & 11.29 \\
\hline & & Mape 1 & 12.63 & 2.10 & 3.05 & 6.02 & 4.07 & 13.90 & 4.00 & 13.19 & 15.36 \\
\hline & & Mape 2 & 8.89 & 2.53 & 3.14 & 7.46 & 4.81 & 14.43 & 6.78 & 18.10 & 11.93 \\
\hline
\end{tabular}

Smape1: stands for the SMAPE obtained by the proposed methodology Smape2: stands for the SMAPE obtained by the original MISMO_CV Mape1: stands for the MAPE obtained by the proposed methodology Mape2: stands for the MAPE obtained by the original MISMO_CV

The values are in percentage

The following table gives the average values for the different quantities and horizon then, and overall average is given:

Table 2. Averaged values

\begin{tabular}{|c|c|c|c|c|c|c|}
\hline \multirow{2}{*}{ Average } & \multicolumn{7}{|c|}{ Horizon } & \multirow{2}{*}{$\begin{array}{c}\text { Total } \\
\text { average }\end{array}$} \\
\cline { 2 - 7 } & 1 & 2 & 3 & 10 & 15 & 6.17 \\
\hline Smape1 & 5.34 & 5.42 & 4.78 & 6.79 & 8.49 & 6.26 \\
\hline Smape2 & 5.66 & 5.23 & 4.71 & 7.08 & 8.60 & 6.18 \\
\hline Mape1 & 5.48 & 5.66 & 4.92 & 6.54 & 8.26 & 6.29 \\
\hline Mape2 & 5.76 & 5.53 & 4.83 & 6.61 & 8.68 & \\
\hline
\end{tabular}

The figure below shows the plots of some predicted (using the newly proposed methodology) values together with the real values 

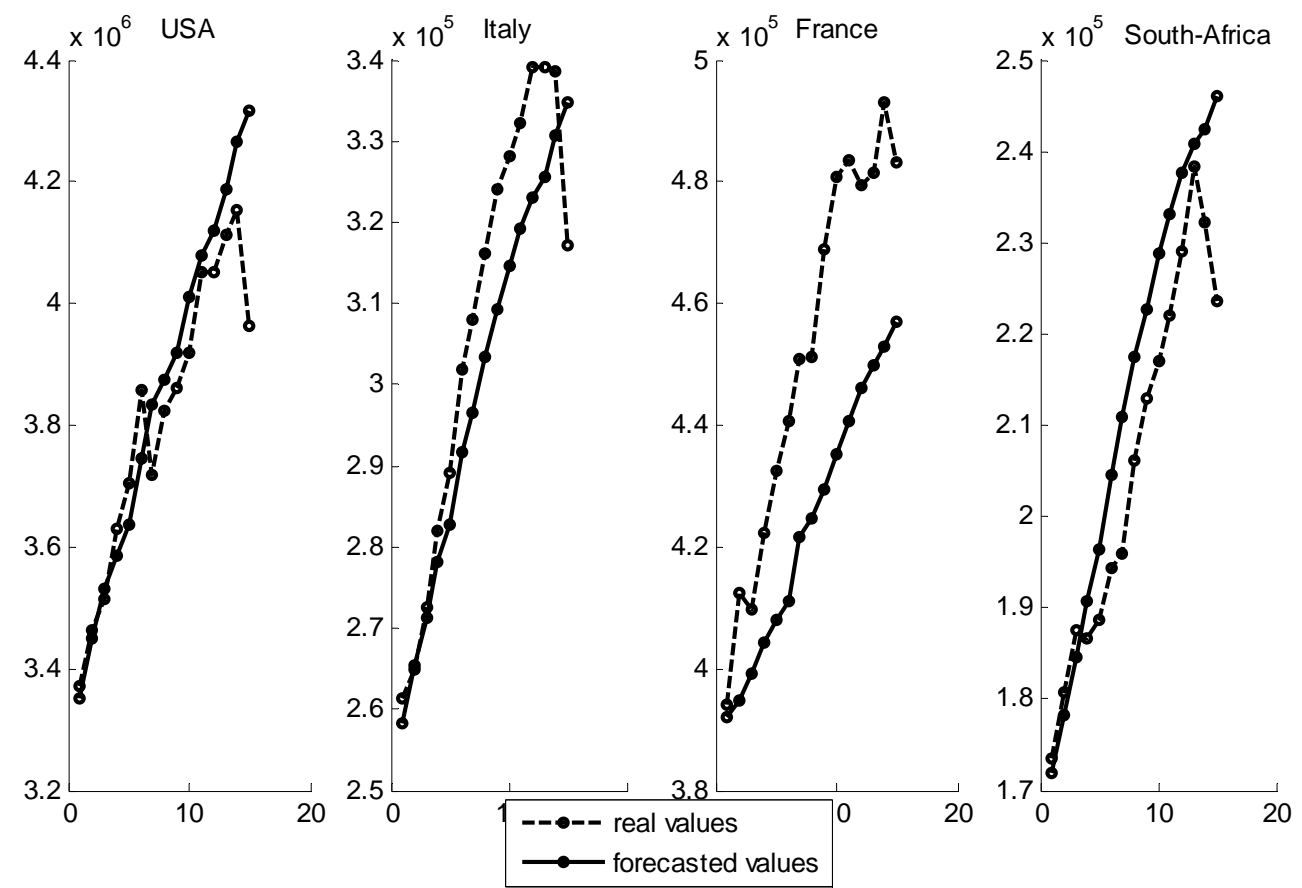

Figure 2. Some forecasted values together with real values for horizon 15

From Table 2, it is seen that, the proposed methodology has the best overall average for horizons 10 and 15. Although the data set was not large enough, it is seen from Table 1 that, these methods can be trusted in forecasting annual electricity load consumption with very good precision. It is also seen from Table 1 that, the overall accuracy is best for the time series with larger datasets (USA, France, Germany, and Italy). This means that the more data we have, the more we are able to predict the future values.

\section{The Cameroonian consumption for horizon 2035}

This part estimates the Cameroonian annual electricity consumption for horizon 2035 when the country aspires to become an emerging economy. An emerging economy needs to have sufficient electricity generation capacity to fuel industrialization and economic growth, as seen in the dataset of the World data bank (an average of 2,000 kWh/inhabitant). It is therefore supposed that in 2035, Cameroon aspires having a consumption of 2,000 kWh/inhabitant. Since the actual consumption is estimated at $287 \mathrm{kWh} / \mathrm{inh}$ abitant, the difference gives 1,713 $\mathrm{kWh} /$ inhabitant. An annual increase of $71.4 \mathrm{kWh} / \mathrm{inh}$ abitant is therefore necessary in order to achieve this goal. 
The population growth from 2012 to 2035 is estimated using the newly proposed methodology. From the population forecast for 2035, the overall annual electricity demand is estimated by multiplying the population by the corresponding consumption per inhabitant. Thereafter, the production capacity of Cameroonian electric power plants in 2035 is estimated by dividing the amount of energy needed from 2012 to 2035 by 8760 , i.e. number of hours per year.

The table below summarizes this section:

Table 3. Cameroonian electricity consumption forecast for 2035

\begin{tabular}{|c|c|c|c|c|c|c|c|c|c|c|c|c|}
\hline Year & 2012 & 2013 & 2014 & 2015 & 2016 & 2017 & 2018 & 2019 & 2020 & 2021 & 2022 & 2023 \\
\hline $\begin{array}{l}\text { Forecasted } \\
\text { Population }\end{array}$ & 20469928 & 20919142 & 21379879 & 21853241 & 22339764 & 22838417 & 23346399 & 23859998 & 24376981 & 24896433 & 25419651 & 25949313 \\
\hline $\begin{array}{c}E \\
(k W h / \text { inhab) }\end{array}$ & 359 & 431 & 503 & 575 & 647 & 719 & 791 & 863 & 935 & 1007 & 1079 & 1151 \\
\hline E (TWh) & 7.35 & 9.02 & 10.76 & 12.57 & 14.46 & 16.43 & 18.47 & 20.60 & 22.70 & 25.08 & 27.43 & 29.87 \\
\hline Year & 2024 & 2025 & 2026 & 2027 & 2028 & 2029 & 2030 & 2031 & 2032 & 2033 & 2034 & 2035 \\
\hline $\begin{array}{l}\text { Forecasted } \\
\text { Population }\end{array}$ & 26489346 & 27041920 & 27607262 & 28183730 & 28769202 & 29360672 & 29955993 & 30554763 & 31156582 & 31759516 & 32361271 & 32960491 \\
\hline E (kWh/hab) & 1223 & 1295 & 1367 & 1439 & 1511 & 1583 & 1655 & 1727 & 1799 & 1871 & 1943 & 2015 \\
\hline E (TWh) & 32.40 & 35.02 & 37.74 & 40.56 & 43.48 & 46.48 & 49.58 & 52.77 & 56.06 & 59.42 & 62.88 & 66.42 \\
\hline
\end{tabular}

Where:

E: energy

kWh/inhab: kilowatthour per inhabitant

TWh: Terawatthour

From Table 3, it is seen that the Cameroonian electricity consumption will rise from 7.35 TWh in 2012 to 66.42 TWh in 2035 . This corresponds to a total increase in electricity consumption of 59.07 TWh. To produce this amount of energy, Cameroon will need a generation capacity of 6.7 GW in 2035. 


\section{Conclusion}

In most cases, electricity demand forecasting is done with the method of scenario building. In this paper various methods for long-, medium- and short-term consumption forecasting are reviewed, in addition to the latter. A new approach for forecasting is developed based on the MISMO_CV strategy. This method uses weighted averages to improve on the level of precision of the method of the nearest neighbors. Results obtained using the new approach are compared to those obtained from the conventional scenario building method. The new approach delivers more precise results.

Finally, the new method is applied specifically to the case of Cameroon, which aspires to become an emerging economy by the year 2035. The results reveal that Cameroon shall need to possess an electricity production capacity of 5,660 MW in 2030 and 6,712 MW in the year 2035, i.e. an average capacity increase of about 300 megawatt per annum. Official figures from Cameroon's Ministry of Energy and Water Resources show however that the electric power demand in 2030 shall be between 1,665 MW and 5,612 MW, depending on the projects realized. The maximum demand here is about 50 MW less than the figure obtained from the new method for that same year. The ministry has not published any figure for the electricity demand in the year 2035. Given the higher level of precision of the new method, it is evident that the actual demand in the year 2035 shall be about 6.7gigawatt, whereas a linear extrapolation of the ministry's figures will deliver a much smaller figure.

\section{References}

[1] COURTOIS, C. MARTIN-BONNEFOUS, M. PILLET. Gestion de production. s.l. : Éditions d'Organisation, Quatrième édition, 2003. ISBN : 2-7081-2986-4.

[2] Biancoa V., Manca O., Nardinia S. Electricity consumption forecasting in Italy using linear regression models. Energy 2009, 34, 1413-1421.

[3] Bihrat ÖNÖZ, Mehmetçik BAYAZIT. The Power of Statistical Tests for Trend Detection. Turkish Journal of Engineering and Environmental Sciences, 27(247-251):434, 2003.

[4] Bontempi, G. Long Term Time Series Prediction with Multi-Input Multi-Output local learning. 2008.

[5] Edigera V, Akarb. SARIMA forecasting of primary energy demand by fuel in Turkey. 2007.

[6] Gianluca Bontempi, Mauro Birttari, Hugues Bersini. Local Learning for Iterated Time Series Prediction.

[7] Hossein Iranmanesh, Majid Abdollahzde, Ethem Alpaydin. Projects in Machine Learning. 22 December 2011. Energies 2012, 5, 1-21; doi:10.3390/en5010001

[8] J.V Ringwood, D. Bofelo. Forecasting Electricity Demand on Short, Medium and Long Time Scales using Neural Networks.

[9] Jung, Pierre. Evolution et prévision de la consommation électrique en France.

[10] Melard, Guy. Initialisation à l'analyse des séries temporelles.

[11] MINEE. PDSE. Vol. 1,2,3,4.

[12] Neil A. Gershenfeld, Andreas S. Weigend. The future of time series. july 1993

[13] Nuorati Maralloo M., Koushki A.R, Lucas C., Kalhor A. Long Term Electrical Load Forecasting via a Neurofuzzy Model.

[14] Philippe Marier, ing. MBA. PRÉVISION DE LA DEMANDE . Québec

[15] Pierre-André Julien, Pierre Lamonde et Daniel Latouche. La méthode des scénarios en prospective. La méthode des scénarios en prospective. Ottawa. 1973-1974. Vol. 51, 2.

[16] Souhaib Ben Taieb, Gianluca Bontempi, Antti Sorjamaa and Amaury Lendasse. LongTerm Prediction of Time Series by combining Direct and MIMO Strategies.In Proceedings of the 2009 IEEE International Joint Conference on Neural Networks,2009.

[17] Steven C. Wheelwright, Spyros Makridakis, Rob J. Hyndman. Forecasting methods and applications. 1998. 
[18] T., Chen. A collaborative fuzzy neural approach for long term load forecasting in Taiwan. Comput. Ind. Eng. 2011, doi:10.1016/j.cie.2011.06.003.

[19] Tahiti, Service de l'Energie de. Bilan prévisionnel, Equilibre offre-demande d'électricité à Tahiti.

[20] Taieb, Souhaib Ben. Prévision de séries Temporelles à Long Terme par Apprentissage Automatique. BRUXELLES : s.n., 2008-2009.

[21] Yongnan JI, JIN Hao, Nima Reyhani, Amaury Lendasse. Direct and Recursive Prediction fo Time series using Mutual Information Selection. In Computational Intelligence and Bioinspired Systems: 8th International Workshop on Artificial Neural Networks, IWANN'05, Vilanova i la Geltra, Barcelona, Spain, volume 3512 of Lecture Notes in Computer Science, pages 1010-1017. Springer-Verlag GmbH, June 8-10 2005.

[22] Zifang Huang, Mei-Ling Shyu. K-NN Based LS-VSM Framework for Long-Term Time series prediction.

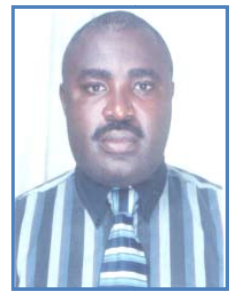

TCHUIDJAN Roger is a senior lecturer of Electrical Power Engineering and High Voltage in the Dept. of Electrical Engineering and Telecommunications in the National Advanced School of Engineering (NASE) of the University of Yaounde I since 1999. He teaches several courses in Power System, High Voltage, Electrical Machines, and Protection of Electrical Power System. He is a former National Director of Electricity with the Gov't of Cameroon. He received a Master's degree in Technical Sciences in 1994, and in 1997 the PhD in Electrical Engineering from the Moscow State University of Transport and Communication. He joined the Power Unit of the Energetics and Automation laboratory of the NASE of Yaounde I university in 1999.

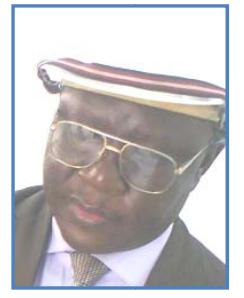

TABE Moses is an Assistant Lecturer and $\mathrm{PhD}$ candidate in the Power Unit of the Energetics and Automation laboratory in the Dept. of Electrical Engineering and Telecommunications in the National Advanced School of Engineering (NASE) of the University of Yaounde I since 2008. He received the Diplomingenieur degree from the German Technological University of Braunschweig in 1988 and a German Master of Business Administration degree in International Economic Relations in 1998. He is currently cumulatively an Inspector of State Procurement in the Presidency of the Republic of Cameroon.

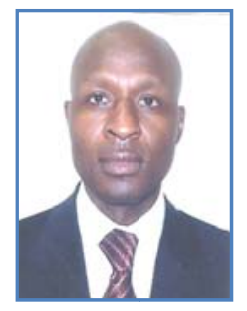

Oumarou HAMANDJODA is holding a $\mathrm{Ph}$. D. in Hydropower Plants and Hydraulics structures, graduated from Moscow Civil Engineering University, Russian Federation. He is an Associate Professor at the National Advanced School of Engineering of Yaounde, Cameroon and equally the Deputy General Manager of AES-SONEL (Integrated Utility). HAMANDJODA is being actively involved in the development of Cameroon energy sector as a whole. This includes, inter alia, the reduction of both technical and non-technical losses in transmission and distribution, integration of the SCADA use in the country energy management system. 


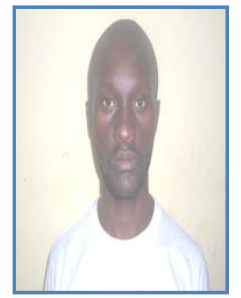

Yannick TOUKE MBELLE is the Head of the Electrical Department of the OYOMABANG THERMAL PLANT of the Integrated Utility AES-SONEL in Cameroon. He received his Diploma of Design Electrical Engineer from the National Advanced School of Engineering, University of Yaounde I in Cameroon in 2012. His research interests are electrical power demand, operation and optimization techniques applied to electrical systems. 